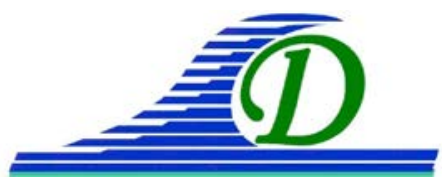

XIII ${ }^{\text {èmes }}$ Journées Nationales Génie Côtier - Génie Civil Dunkerque, 2-4 juillet 2014

DOI:10.5150/jngcgc.2014.094 C Editions Paralia CFL

disponible en ligne - http://www.paralia.fr - available online

\title{
Suivi environnemental des exploitations de granulats marins au large de la pointe de Chassiron. \\ Contribution à la connaissance de la dynamique sédimentaire dans la zone prélittorale
}

\author{
Patrice WALKER $^{1}$, Morgane RAVILLY ${ }^{1}$, Ronan LAUNAY ${ }^{1}$ \\ 1. CREOCEAN, rue Charles Tellier, 17000 La Rochelle, France. \\ creocean@creocean.fr
}

\section{Résumé :}

Reconnu et décrit par le CNEXO en 1977, le gisement de granulats marins de "Chassiron" est situé à une dizaine de km au NW de la pointe éponyme, extrémité septentrionale de l'île d’Oléron.

Il est exploité depuis 1984 et 5 concessions sont actuellement en activité.

Le suivi environnemental réglementaire comprend un levé bathymétrique et une cartographie morphosédimentaire sur une zone d'environ $60 \mathrm{~km}^{2}$ comprenant l'ensemble des concessions, et s'étendant jusqu'aux roches entourant la pointe audessus de l'isobathe $-15 \mathrm{~m} \mathrm{CM}$.

Cette reconnaissance acoustique est complétée par un suivi biosédimentaire basé sur les analyses granulométriques et faunistiques d'échantillons prélevés à la benne en 10 stations caractéristiques.

Les données acquises en 2002, 2006 et 2012 permettent de décrire précisément l'évolution de la couverture de sédiments meubles superficiels et de caractériser la dynamique de la fraction sablo-graveleuse.

Ces mesures confirment que les impacts des prélèvements sont essentiellement confinés à la zone exploitée, et mettent en évidence une grande stabilité des accumulations sédimentaires adjacentes dont les caractéristiques morphologiques restent identiques sur la période d'observation.

Les évolutions observées s'avèrent enfin, tout à fait conformes aux effets prédits par la modélisation hydrosédimentaire réalisée en 2006.

Mots-clés: Granulats marins, Dynamique sédimentaire, Domaine prélittoral, Bathymétrie, Sonar à balayage latéral, Environnement littoral, Ecosystèmes côtiers.

\section{Introduction}

La production annuelle de granulats marins en France est actuellement de 7 millions de tonnes et représente 1,5 à $2 \%$ de la quantité totale des granulats produits, qui a varié entre 350 et 450 millions de tonnes au cours des deux dernières décennies (IFREMER, 2006 et UNPG, 2013). 


\section{Thème 6 - Gestion durable des zones littorales et estuariennes}

Les matériaux extraits sont principalement des sables et graviers siliceux accumulés au fond des paléovallées creusées par les fleuves à travers le plateau continental au cours des périodes glaciaires du Quaternaire.

Le gisement de Chassiron (figure 1) est ainsi localisé dans la vallée incisée par la Charente à travers le seuil interinsulaire, relief sous-marin constitué de roches calcaires qui barre l'entrée du pertuis d'Antioche entre les îles de Ré au Nord et d'Oléron au Sud (CRESSARD \& AUGRIS, 1977 ; WEBER, 2004 ; WEBER et al., 2004).

La géométrie du gisement et les caractéristiques sédimentologiques des matériaux accumulés ont été déterminées en 1975 et 1976 par le CNEXO et le BRGM qui ont mené successivement deux campagnes de prospection par si.mique réflexion marine continue (300 km de profils) puis une campagne de 30 carottages avec un échantillonnage des sédiments superficiels sur, en moyenne, les 4 premiers mètres de profondeur (IFREMER, 2006).

Un premier dossier de demande d'exploitation de sables et graviers mains est alors déposé en 1982 et le titre minier (permis d'exploitation) est accordé par l'Etat en 1984 permettant ainsi le début des extractions de granulats sur le site.

Une deuxième concession est attribuée en octobre 1999 et son exploitation débutera en 2001.

Suite au dépôt d'une troisième demande de permis, l'Etat impose aux concessionnaires et pétitionnaires une reconnaissance détaillée des fonds marins pour établir un état de référence des sites d'extraction et de la zone adjacente, jusqu'à la limite d'affleurement des platiers calcaires prolongeant en domaine subtidal la pointe de Chassiron, extrémité NW de l'île d'Oléron.

Cette campagne est réalisée par la société Alidade entre juillet 2001 et février 2002. Elle comprend une cartographie bathymétrique et morphosédimentaire des fonds par sondeur et sonar à balayage latéral, et une étude biosédimentaire des sédiments superficiels prélevés par benne sur une quarantaine de stations (ALIDADE, 2002).

Par ailleurs, les arrêtés préfectoraux d'ouverture des travaux imposent dès lors aux exploitants de procéder au suivi environnemental de la même zone tous les 5 ans.

La société CREOCEAN a donc procédé à ces travaux de suivi en 2006 et 2011 (CREOCEAN, 2006 \& 2012).

Ce corpus cartographique, unique en terme de superficie et de fréquence des levés apporte des connaissances précises sur l'évolution de la couverture de sédiments meubles superficiels de ce domaine prélittoral soumis à des conditions hydrodynamiques intenses générant une mobilité pratiquement permanente de la fraction sableuse ( IDIER et al., 2006).

Ces résultats sont, de plus, complétés par des données issues d'une reconnaissance morphosédimentaire à très haute résolution, d'un site de $0,5 \mathrm{~km}^{2}$, retenu pour l'implantation expérimentale de récifs artificiels, et localisé à environ $5 \mathrm{~km}$ au Sud de la zone de suivi (CREOCEAN \& MESURIS, 2012.) Cette cartographie acoustique à très 


\section{XIII ${ }^{\text {èmes }}$ Journées Nationales Génie Côtier - Génie Civil \\ Dunkerque, 2-4 juillet 2014}

haute résolution par sondeur multifaisceaux nous renseigne notamment sur la géométrie précise des accumulations sédimentaires en rubans et sillons observées fréquemment sur la zone de suivi.

Les évolutions bathymétriques et morphosédimentaires de la couverture sédimentaire seront par ailleurs comparées aux tendances au dépôt ou à l'érosion, prédites par modélisation hydrosédimentaire.

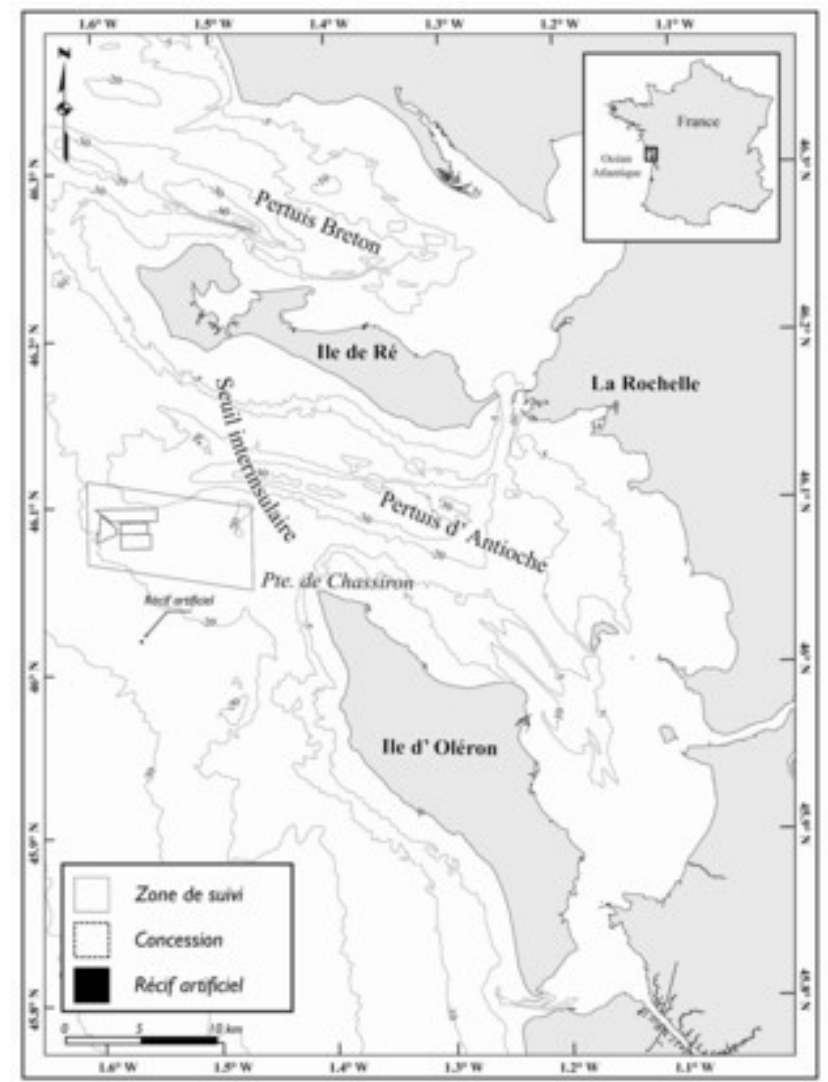

Figure 1. Localisation des concessions de Chassiron.

\section{Données et méthodes}

Le suivi environnemental des exploitations est précisément défini par l'annexe II des arrêtés préfectoraux d'ouverture des travaux et doit être effectué tous les 5 ans sur la zone de $60 \mathrm{~km}^{2}$ indiquée figure.1.

\subsection{Cartographie acoustique : bathymétrie, nature des fonds}

Le contrôle bathymétrique est effectué par sondeur vertical monofaisceau monofréquence (200 kHz) en 2002 puis bifréquence (33 et $200 \mathrm{kHz}$ ) en 2006 et 2011.

Les données géophysiques ont été collectées le long de profils longitudinaux (profils d'azimut $\mathrm{N} 100^{\circ}$, parallèles au grand bord de la zone cartographiée et espacés de $175 \mathrm{~m}$ ) complétés par des profils transversaux (profils d’azimut N0², espacés de $350 \mathrm{~m}$ ). 


\section{Thème 6 - Gestion durable des zones littorales et estuariennes}

Le maillage est identique pour l'ensemble des levés ce qui permet une description fine des caractéristiques des fonds marins et facilite l'approche comparative afin de caractériser l'évolution des profondeurs sur une décennie. La fréquence d'acquisition des sondes est de $1 \mathrm{~Hz}$, soit en moyenne la restitution d'un point sondé tous les $2 \mathrm{~m}$ le long des profils, et un total d'environ 200000 sondes. La méthode de réduction des sondes au zéro des cartes marines, utilisée lors de la reconnaissance de 2002 ne prenait pas en compte la mesure de marée sur site puisque la correction appliquée est la marée prédite au port de La Pallice. Le protocole mis en œuvre en 2006 et 2011 est beaucoup plus fiable puisque la mesure de sonde est rattachée au système altimétrique NGF par une mesure altimétrique en temps réel, de la base acoustique du sondeur en utilisant un système GPS RTK avec une précision centimétrique. Cette méthode garantit une excellente répétitivité des mesures et réduit sensiblement les incertitudes. La précision des profondeurs restituées est ainsi d’ordre décimétrique pour les campagnes 2006 et 2011. La cartographie de la nature des fonds marins repose principalement sur l'acquisition d'images acoustiques de haute fréquence par sonar à balayage latéral avec une résolution spatiale d'ordre décimétrique (BERNE et al., 1986). Les caractéristiques acoustiques des sonars utilisés en 2002 et 2006 sont identiques et le sonar Klein 3000 mis en oeuvre en 2011 est plus performant puisqu'il permet l'acquisition simultanée de signaux bifréquence (100 et $500 \mathrm{kHz}$ ) et donc de données de plus haute résolution sur des objets particuliers si nécessaire. Par ailleurs, les signaux rétrodiffusés sont convertis en données numériques dans l'électronique remorquée (poisson) ce qui garantit une acquisition plus exhaustive et facilite les traitements ultérieurs des images. Les images acoustiques sont précisément géoréférencées ce qui permet de procéder à un assemblage sous forme de mosaïque (figure 2).

Ces images en niveaux de gris sont ensuite interprétées afin de restituer les caractéristiques morphosédimentaires des fonds :

- des teintes claires traduisent des fonds faiblement rétrodiffusants et marquent la présence defonds sédimentaires de fine granulométrie ou d'ombres acoustiques,

- à l'inverse, des teintes foncées marquent la présence de fonds présentant un fort coefficient de rétrodiffusion, constitués par des matériaux sédimentaires grossiers, des fonds rocheux et/ou des reliefs positifs.

Au-delà de la nature des fonds, les données d'imagerie permettent d'appréhender la dynamique de la couverture sédimentaire meuble au travers de la présence éventuelle de figures sédimentaires.

Les faciès acoustiques sont également calibrés par l'analyse granulométrique d’échantillons caractéristiques prélevés à l'aide d’une benne Smith-Mac Intyre. 


\section{XIII ${ }^{\text {èmes }}$ Journées Nationales Génie Côtier - Génie Civil \\ Dunkerque, 2-4 juillet 2014}

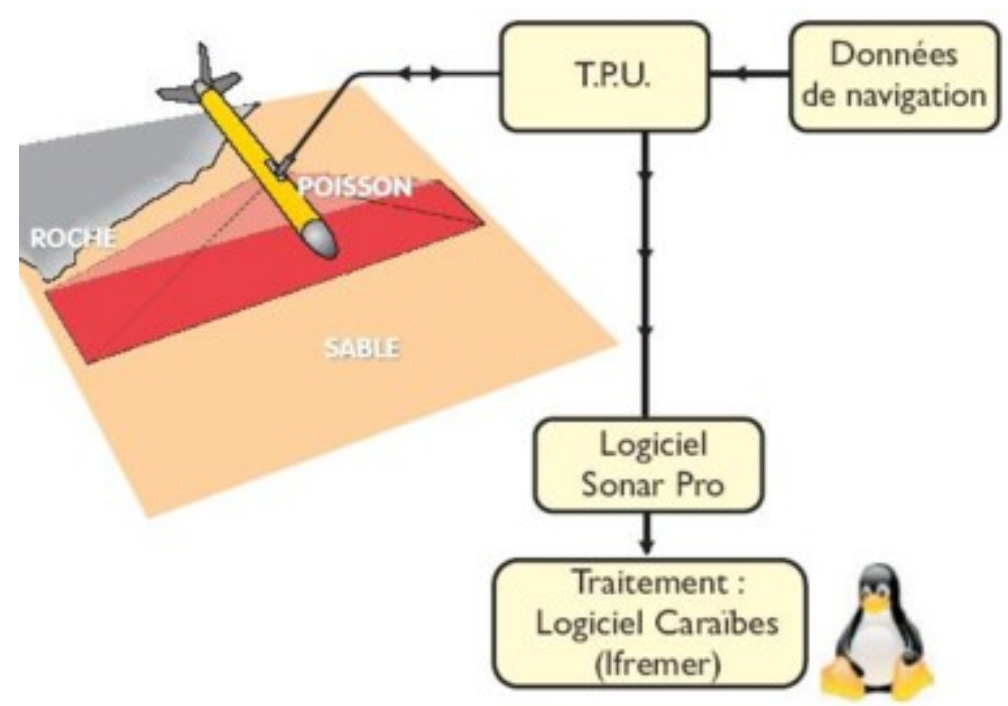

Figure 2. Chaine d'acquisition et de traitement des données de sonar à balayage latéral.

\subsection{Analyses biosédimentaires et évolution des peuplements benthiques}

10 stations caractéristiques sont échantillonnées depuis 2002 afin de récolter les sédiments et la macrofaune benthique associée sur une surface d'au moins $0,5 \mathrm{~m}^{2}$.

Les espèces sont ensuite déterminées selon la nomenclature donnée par l'European Register of Marine Species (ERMS). Les données statistiques permettent ensuite de caractériser les peuplements en terme d'abondance et de densité.

2.3 Données morphobathymétriques acquises sur le site projeté pour l'installation de récifs artificiels.

Elles comprennent également des données de sonar à balayage latéral étalonnées par des prélèvements mais les données bathymétriques ont été acquises avec la vedette Survex 1 de la société Mesuris, équipée d’un sondeur multifaisceaux Seabat Reson.

\subsection{Modélisations hydrosédimentaires.}

Les impacts des surcreusements engendrés par les extractions ont été évalués en 2006 par simulation numérique mise en œuvre en utilisant :

- le code Telemac 2D développé par LNH EDF. pour calculer les conditions de niveau et les courants de marée,

- et le code SWAN (TU Delft) pour déterminer les conditions d'agitation.

Les forçages de courant et de houle ont ensuite été utilisés pour calculer les tendances au transport à l'aide du code TRANSPOR 1993 proposé par Van Rijn.

Les tendances à l'accrétion ou à l'érosion sont alors estimées en fonction de la divergence du vecteur "transport solide". 


\section{Résultats}

\subsection{Cartographie morphosédimentaire et évolution des fonds (figure 3)}

Des zones de hauts-fonds rocheux fortement structurés occupent la partie orientale de la zone prospectée (en gris sur la figure) et se caractérisent par des profondeurs pouvant atteindre $16 \mathrm{~m} \mathrm{CM}$.

Sur les $90 \%$ de la zone prospectée, les fonds sont associés à une couverture de formations sédimentaires meubles constituée par une semelle de matériaux grossiers (graviers / sables grossiers) figurée en rouge sur la carte et recouverte de sables moyens et fins, représentés en jaune. La mobilité de la couverture superficielle est attestée par la présence de sillons, $\mathrm{N} 80^{\circ}$ à $\mathrm{N} 140^{\circ}$, reflétant des écoulements secondaires dans la couche limite et la présence de mégarides (tildes bleus) qui semblent indiquer, tout au moins au SE de la zone prospectée, un sens du transport résiduel du SW vers le Pertuis d'Antioche.

Les grandes unités morphosédimentaires sont remarquablement stables sur la décennie et les modifications les plus significatives sont cantonnées aux zones concédées en raison des dragages d'exploitation. A l'extérieur de ces périmètres, les limites des accumulations de sables fins à moyens au-dessus des roches, et des affleurements de graviers, restent constantes. Les figures sédimentaires associées ne varient pas significativement ce qui confirme une grande stabilité des mouvements sédimentaires orientés majoritairement du SW vers le NE (figures 4 et 5).

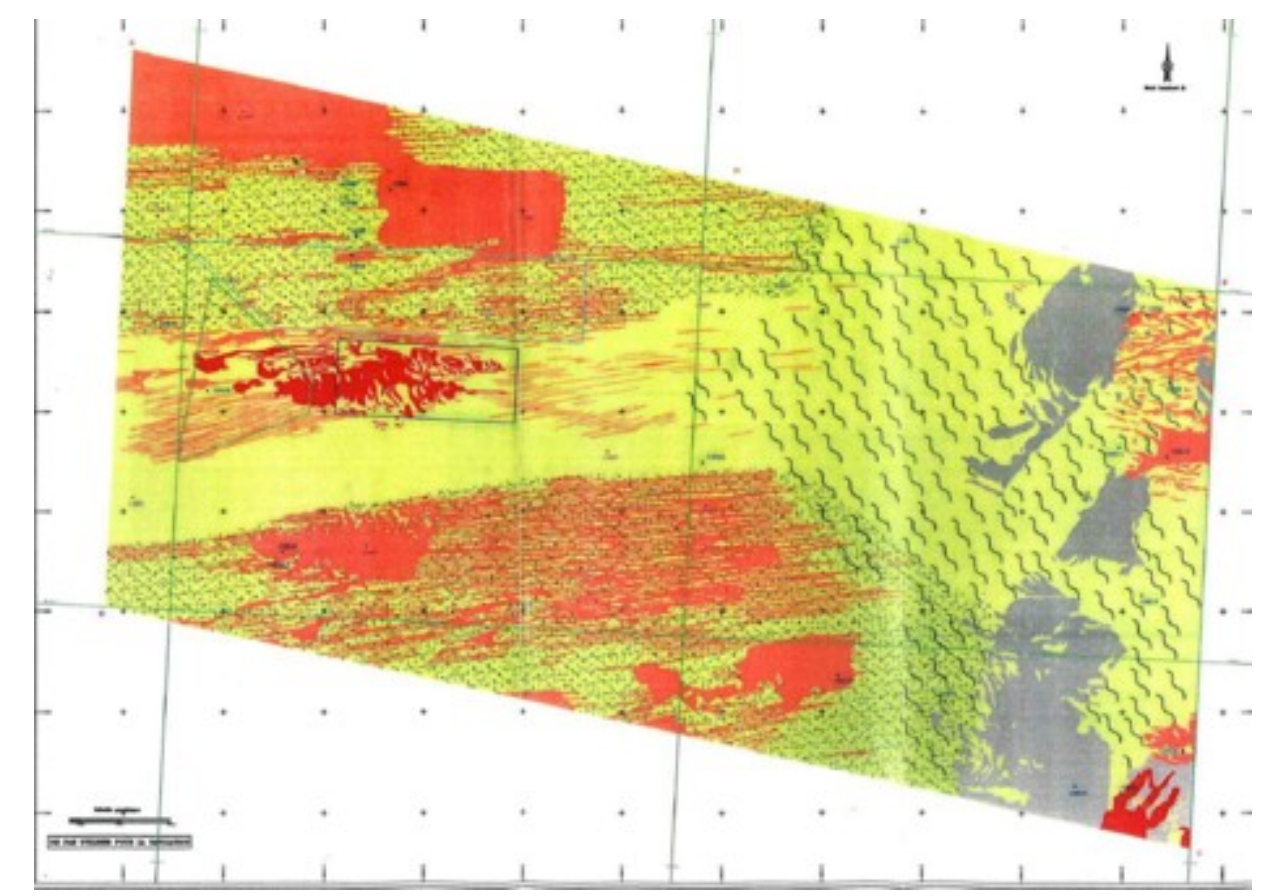

Figure 3. Etat de référence du gisement de Chassiron - Carte des formations superficielles (ALIDADE, 2002). 


\section{XIII ${ }^{\text {èmes }}$ Journées Nationales Génie Côtier - Génie Civil \\ Dunkerque, 2-4 juillet 2014}

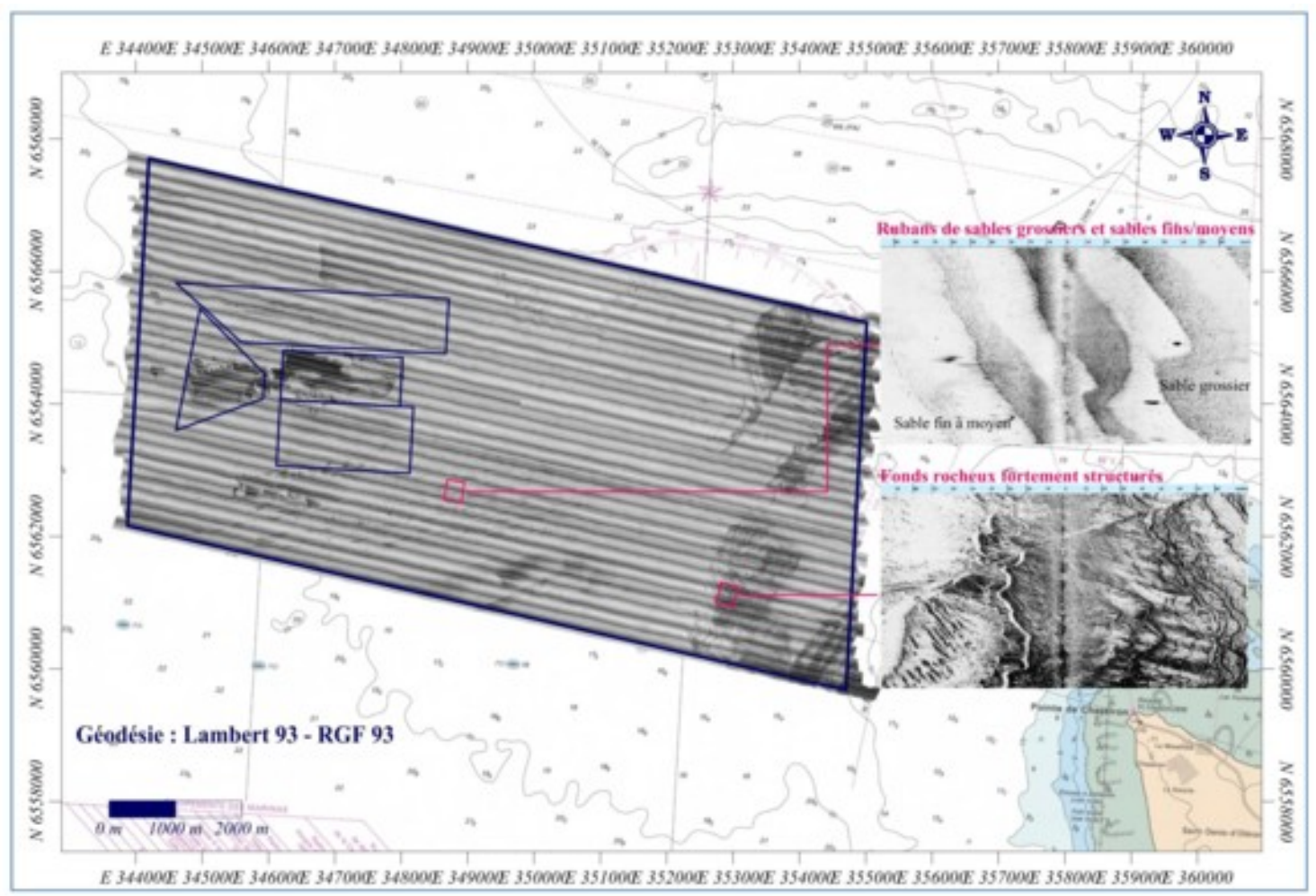

Figure 4. Suivi quinquennal 2011 des concessions de Chassiron. Imagerie acoustique.

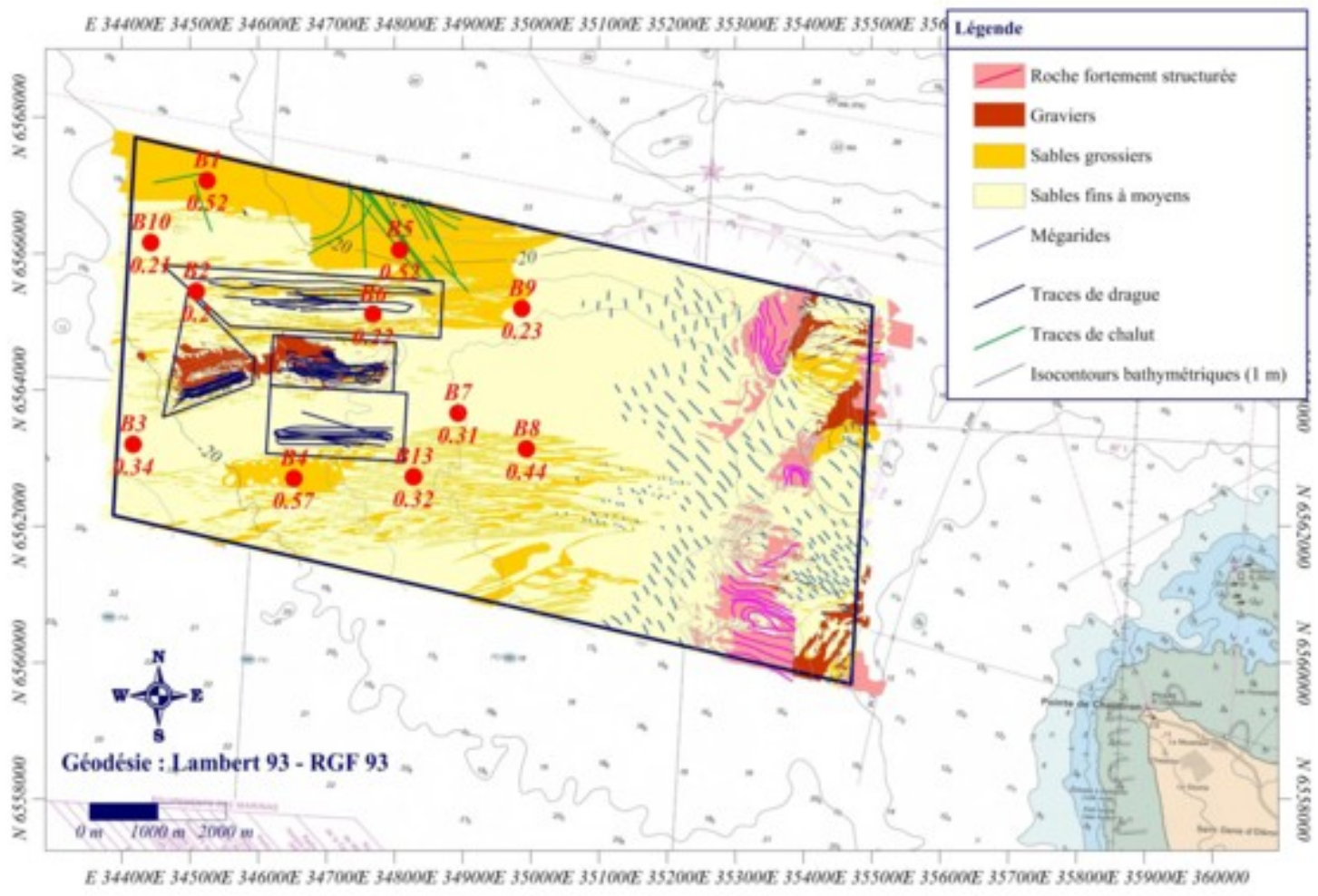

Figure 5. Suivi quinquennal 2011 des concessions de Chassiron, nature des fonds. 


\section{Thème 6 - Gestion durable des zones littorales et estuariennes}

La comparaison des données bathymétriques entre 2006 et 2011 confirme totalement la cartographie acoustique puisque les variations bathymétriques dépassent rarement 0,5 m hormis sur la zone exploitée (figure 6). A l'Est de la zone de suivi, les mouvements sédimentaires en bordure du platier rocheux sont plus marqués avec une prépondérance des zones en accumulation sur les zones en érosion.

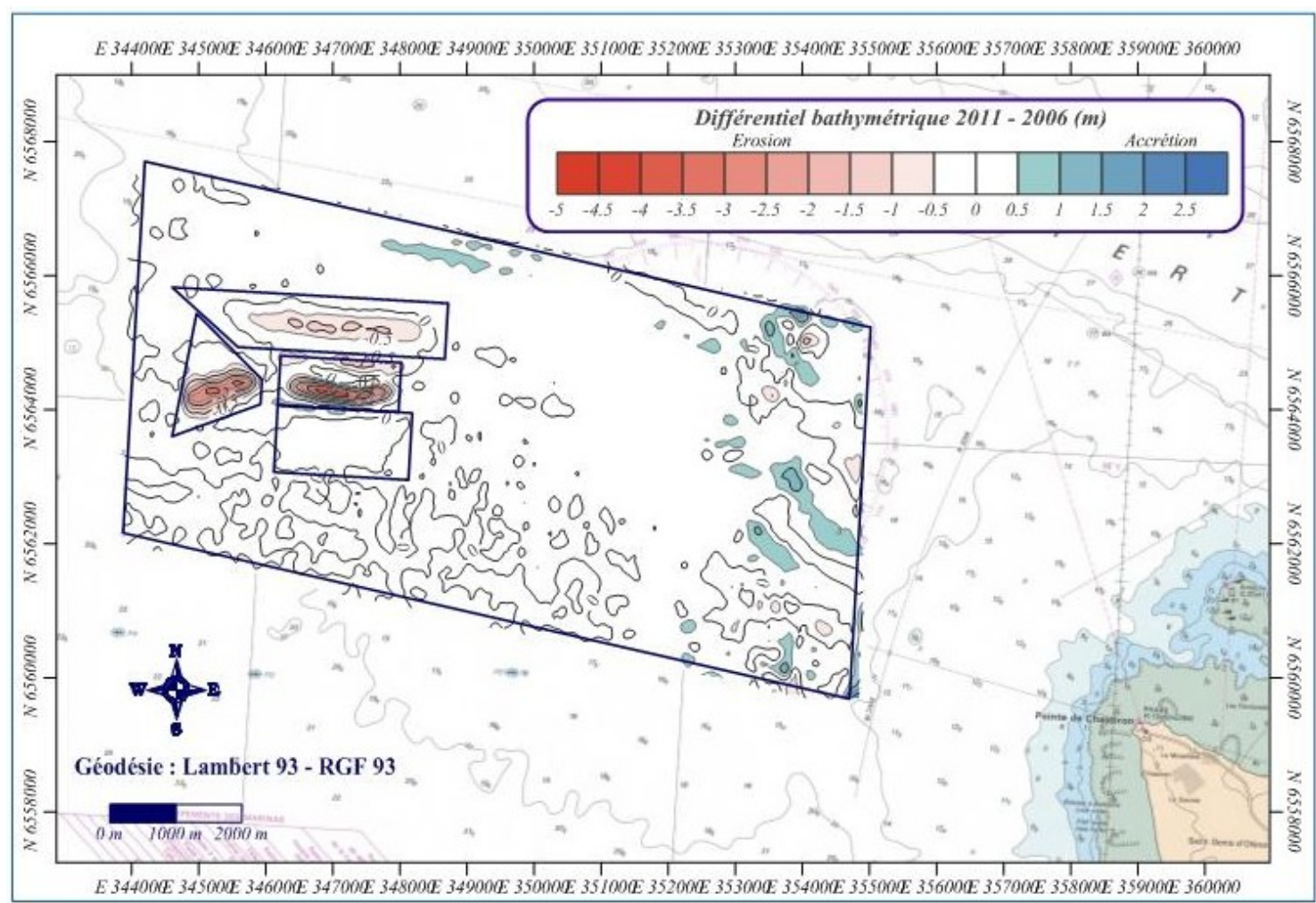

Figure 6. Différentiel bathymétrique 2006-2011du suivi des concessions de Chassiron.

Les données bathymétriques acquises en 2012 sur la zone d'implantation future de récifs artificiels nous enseignent enfin que les amplitudes des sillons et des mégarides observés sur les accumulations sableuses sont d’ordre décimétrique.

La comparaison des profondeurs réduites au 0 CM entre 2002 et 2011 ne révèle pas de différences significatives sauf naturellement au droit des zones exploitées avec un approfondissement maximum de l'ordre de 4 m mais les incertitudes cumulées liées aux mesures sont probablement d'ordre métrique.

A l'Est de la zone exploitée, les profondeurs relevées en 2012 sur les accumulations de sables fins à moyens, semblent plutôt moins importantes qu'en 2002 traduisant ainsi une tendance à l'accumulation des sédiments sur le flanc occidental du seuil interinsulaire.

Cette tendance avait été également observée sur les cartes issues des simulations numériques des tendances au transport sableux et au dépôt/érosion ((figure 7). Ces 


\section{XIII ${ }^{\text {èmes }}$ Journées Nationales Génie Côtier - Génie Civil \\ Dunkerque, 2-4 juillet 2014}

simulations prenaient également en compte un approfondissement maximum théorique de 5 m sur l'ensemble de la zone concédée en raison des dragages.
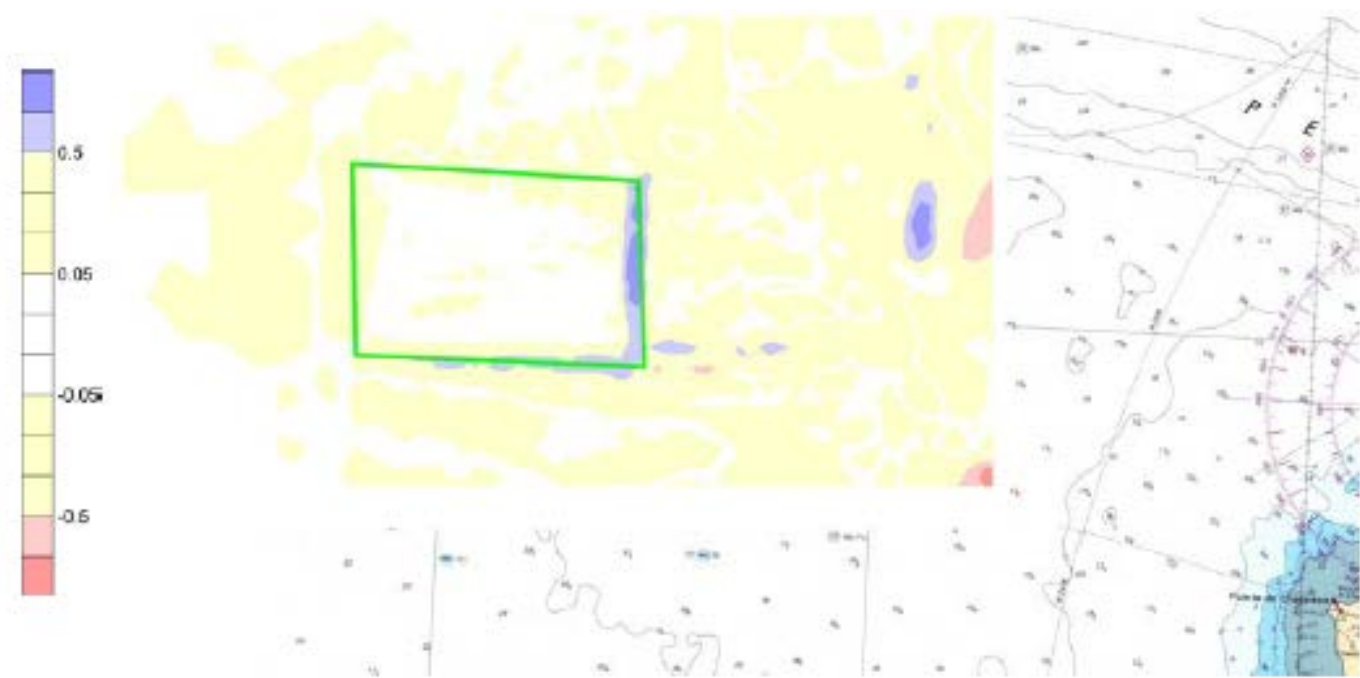

Figure 7. Taux d'érosion/sédimentation annuel (en m) simulé pour des sables de $500 \mu \mathrm{m}$.

\section{Conclusions}

Ce suivi décennal confirme que les impacts des extractions sont tout à fait conformes à ceux prévus par les études préalables à l'ouverture des travaux. La morphologie des accumulations et les figures sédimentaires observées mettent en évidence la grande mobilité des sédiments meubles sur le seuil interinsulaire mais cette dynamique sédimentaire intense se traduit vraisemblablement par un transit net des matériaux du large vers le pertuis d'Antioche, processus qui n'est en rien affecté jusqu'à maintenant par les prélèvements de matériaux sur les exploitations.

\section{Remerciements}

Nous remercions les sociétés concessionnaires, du site de Chassiron le CRPMEM Poitou-Charentes et le CREAA d'avoir accepté de publier en commun les résultats de leurs campagnes à la mer respectives.

\section{Références bibliographiques}

ALIDADE (2002). Etat de référence environnemental du gisement de sables et graviers siliceux de Chassiron. Rapport et annexes, Brest, 38 p.

BERNE S., AUGUSTIN J.M., BRAUD F., CHENE G., WALKER P. (1986). Cartographie et interprétation de la dynamique sédimentaire des plates-formes 
continentales : améliorations de la technique d'observation par sonar latéral. Bull. Soc. Géol. France, Vol. 8(2), pp 437-446.

CREOCEAN (2006). Gisements de granulats marins de CHASSIRON. Suivi quinquennal - Etat 2006. Rapport et annexes, Créocéan n¹062042, La Rochelle, 21 p. CREOCEAN (2012). Gisements de granulats marins de CHASSIRON. Suivi quinquennal - Etat 2011. Rapport et annexes, Créocéan n¹1107, La Rochelle, 70 p. CREOCEAN, MESURIS (2012), Campagne de reconnaissance géophysique des fonds marins sur le périmètre projet d'immersion de récifs artificiels expérimentaux en Charente-Maritime, pour le compte du CRPMEM Poitou-Charentes et du CREAA. Cartes et rapport. 17 p.

CRESSARD A.P., AUGRIS C. (1977). Recherche de granulats marins pour l'approvisionnement de la région Poitou-Charentes. Rapport CNEXO-Centre Océanologique de Bretagne, Brest, 54 p.

IDIER D., PEDREROS R., OLIVEROS C., SOTTOLICHOIO A., CHOPIN L., BERTIN X. (2006). Contributions respectives des courants et de la houle dans la mobilité sédimentaire d'une plateforme interne estuarienne. Exemple: le seuil interinsulaire, au large du Pertuis d'Antioche, France. C. R. Geosciences, Vol. 338, pp 718-726. http://dx.doi.org/10.1016/j.crte.2006.05.004

IFREMER (2006) Inventaire des ressources en matériaux marins. Façades "MancheEst et" et "Loire-Gironde". AUGRIS C. Coord. 165 p.

UNPG -Union Nationale des Producteurs de Granulats- (2013). L'industrie française des granulats en 2012. Disponible en ligne sur : http://www.unpg.fr/tpl/download.php?id=2067

WEBER N. (2004). Morphologie, architecture des dépôts, évolution séculaire et millénaire du littoral charentais. Thèse de doctorat, Université de La Rochelle, 289 p.

WEBER N., CHAUMILLON E., TESSON M., GARLAN T. (2004), Architecture and morphology of the outer segment of a mixed tide and wave-dominated-incised valley, revealed by HR seismic reflection profiling: the paleo-Charente River, Marine Geology, 207(1-4), pp 17-38. http://dx.doi.org/10.1016/j.margeo.2004.04.001 